\title{
Amplitude and timing jitter reduction using a fiber NOLM incorporating a fiber Bragg grating based pulse shaper
}

\author{
F. Parmigiani, P. Petropoulos, P.J. Almeida, M. Ibsen, D.J. Richardson \\ Optoelectronics Research Centre, University of Southampton, Southampton SO171BJ, UK
}

\begin{abstract}
:
We demonstrate the simultaneous removal of amplitude and timing jitter from an RZ data-stream using a superstructured fiber grating pulse shape converter and subsequent gating of a clean optical clock in a NOLM.
\end{abstract}

\section{Introduction}

Superstructure fiber Bragg grating (SSFBG) technology allows the fabrication of filters with an almost arbitrary spectral response. SSFBGs rely on the capability to precisely vary the spatial refractive index modulation during the fabrication of the grating. Such grating filters can be useful for a variety of applications in which accurate spectral control both in amplitude and in phase is required. In the past we have demonstrated the use of SSFBGs for the reshaping of ultrashort pulses. An interesting application of this linear optical technique relates to the generation of rectangular pulses of a few ps duration [1] which are difficult to generate by any other means such as direct electrooptic modulation. Such pulse forms can be very useful for applications that involve nonlinear optical switching of short data pulses. We have already demonstrated the benefit of using such pulses in a timing-jitter-tolerant demultiplexing scheme operating at repetition rates of up to $80 \mathrm{Gbit} / \mathrm{s}[2,3]$. This scheme used shaped rectangular pulses as the control signal to a nonlinear switch based on a nonlinear optical loop mirror (NOLM). The correspondingly broad switching window (relative to the pulses to be demultiplexed) relaxed the tolerance on the relative timing between the data and the clock although it is to be appreciated that the original jitter itself was not eliminated and was passed on to the demultiplexed signal. In a more recent experiment [4], we shaped the data itself rather than the control signal before feeding it into the NOLM. This allowed us not only to have timing-jittertolerant operation of the switch but also to retime the data pulses at the switch output. In this paper we extend this previous work and obtain pulse reshaping/amplitude noise reduction as well as retiming of the data pulses in a single step using the regenerative properties of a NOLM. Our technique relies upon converting the incident data pulses into rectangular pulses at the NOLM input and using these broadened pulses to switch a well-conditioned and welldefined local clock signal. The S-shaped switching characteristic of the NOLM and square switching window provide the pulse reshaping and amplitude noise reduction. In the current work we have artificially introduced timing jitter and amplitude noise however this scheme should ultimately prove useful for 3R signal regeneration in ultra-high bit rate long-haul transmission systems. We demonstrate the improvement in the noise performance of our system through both bit-error rate (BER) and eye diagram measurements.

\section{Principle of operation and experimental set-up}

The experimental set-up and principle of operation of our reshaping-retiming scheme is shown in Fig.1a. A $10 \mathrm{GHz}$ actively mode-locked erbium fiber ring laser (EFRL) was used as the data source to generate $\sim 2.5$ ps soliton pulses. The wavelength of operation of the laser was tuned to $1556.8 \mathrm{~nm}$. The laser included a phase-lock-loop-controlled servo mechanism to continuously adjust the cavity length so as to ensure that stable locking was maintained at the required pulse repetition frequency. The pulses were modulated to provide a $2^{31}-1$ pseudorandom data sequence using a lithium niobate modulator and were then fed onto the pulse-shaping SSFBG (via a circulator) to convert the $2.5 \mathrm{ps}$ solitons into $20 \mathrm{ps}$ rectangular pulses. The shaped data pulses were used as the control signal to a NOLM. The nonlinear medium in the NOLM consisted of 220m of Highly Nonlinear Fiber (HNLF) with a nonlinear parameter of $\gamma=\sim 20 \mathrm{~W}^{-1} \mathrm{~km}^{-1}$ and a zero-dispersion wavelength at $1550 \mathrm{~nm}$. It is known that a suitably biased NOLM has a nonlinear transfer characteristic and is able to reduce the noise both on the zeros and the marks of the control signal, provided that the peak power of the control pulses reaches the value required to achieve a $\pi$ nonlinear phase shift over the length of the fiber inside the loop [5]. The transfer characteristic of the NOLM used in our experiments is shown in Fig. 1b, and the shaped data signal was amplified sufficiently to ensure that the desired operating point was reached. 


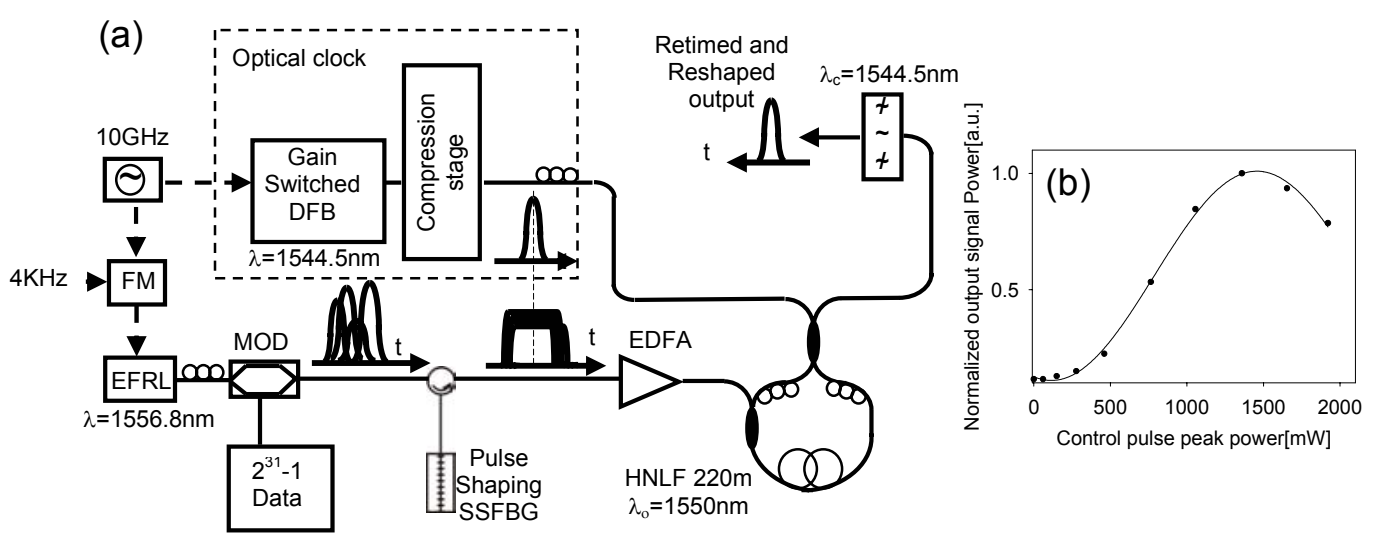

Figure $1 \mathrm{a}$ : Experimental setup and principle of retiming and reshaping operation, b: nonlinear transfer characteristic of the NOLM

The input signal to the NOLM was provided by a gain-switched Distributed Feedback (DFB) laser, which operated at $1544.5 \mathrm{~nm}$ and was driven by a $10 \mathrm{GHz}$ RF signal synchronized to the laser oscillator. The pulses of the gain-switched DFB laser were compressed down to $\sim 2$ ps using a $125 \mathrm{~m}$ of dispersion compensating fiber followed by a nonlinear compression stage comprising an EDFA and 490m of a HNLF.

In order to study the regenerative properties of our system, we artificially introduced amplitude and timing jitter to the data pulses. The timing jitter was introduced by frequency modulating the RF drive signal of the EFRL with a $4 \mathrm{kHz}$ tone. The frequency of the modulating signal was intentionally kept low in order to allow the phase-lock loop of the laser to follow the changes in applied RF frequency. In addition to this, we degraded the extinction ratio between the marks and spaces of the data by choosing a non-optimum bias voltage for the operation of the lithium niobate data modulator. This enabled us to degrade the Q-factor of the data eye diagram from 17.5 down to 7.4.

\section{Results}
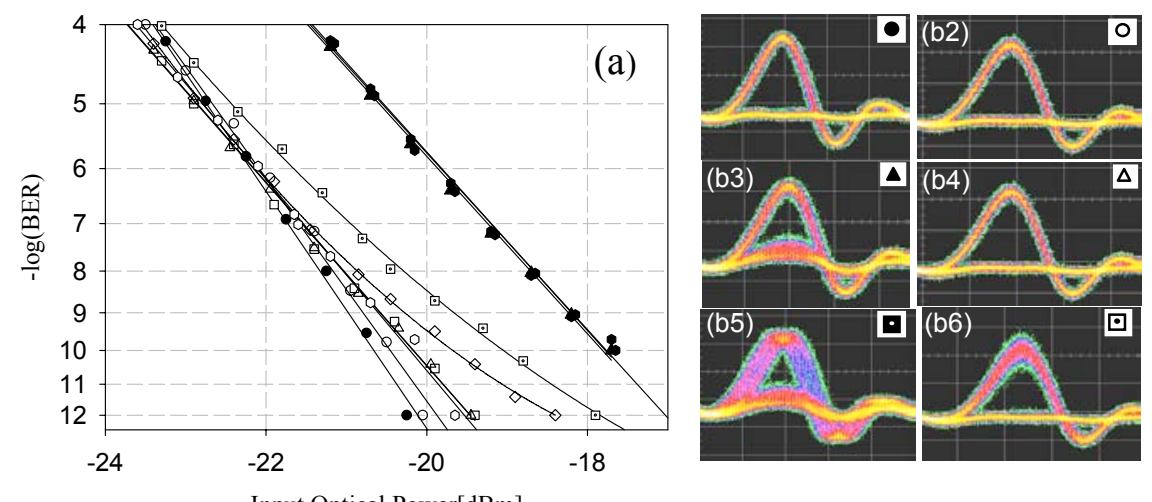

O Back to back
No timing jitter and induced amplitude jitter
$\triangle \sim 0.8 p s$ induced timing jitter and induced amplitude jitter
Full-symbols: before the system, Open-symbols: after the system

Figure 2 BER curves and eye diagrams at various positions and settings of the system.

In order to evaluate the performance of our system we first examined the switched signal for the case that no timing or amplitude jitter was applied to the data pulses. The eye diagrams in Fig.2b1 and Fig.2b2 correspond to the data signal at the input and the output of the system respectively, and the BER curves demonstrate that the retiming and reshaping system does not in itself introduce any additional noise to the signal. We next examined the quality of the output signal for the case when just amplitude noise was deliberately applied to the incident data pulses. An improvement in the receiver sensitivity of more than $2.3 \mathrm{~dB}$ was achieved after the switch, demonstrating the amplitude noise reduction provided by the NOLM. 
The system performance was assessed next for the case when timing jitter was added together with amplitude noise onto the input data. The amount of induced timing jitter could be varied by varying the amplitude of the frequency modulation applied to the $10 \mathrm{GHz}$ laser drive signal. Note that by induced timing jitter we imply the amount of pulse period fluctuation as defined by the amplitude of the frequency modulating noise signal. Obviously, the timing jitter of the pulse source itself has to be considered in addition to the induced timing jitter to calculate the corresponding total timing jitter. For our measurements we considered the same constant amount of amplitude noise and five different values of induced timing jitter. Starting from the case where very little timing jitter was induced $(\sim 0.8 \mathrm{ps})$ we finally considered the most extreme case of induced timing jitter $(\sim 11 \mathrm{ps})$, to provide a significant test of our system. As shown in Fig.2b, the timing jitter is eliminated at the output of the system and open eye diagrams are obtained in all cases. In the case of $\sim 5.5 \mathrm{ps}$ induced timing jitter the BER curve still overlaps with the back-to-back curve. For the most extreme cases, slight amplitude noise and a small power penalty in the BER curves $(\sim 1 \mathrm{~dB}$ for $11 \mathrm{ps}$ induced timing jitter) are observed. We suspect that some of this noise may be introduced due to the nonlinear distortion caused by self-phase modulation experienced by the clock/data signal during propagation in the HNLF.

It is also worth noting that the BER curves of the input signal do not show any degradation with increasing timing jitter. This is clearly understandable due to the low-pass filtering action of the $10 \mathrm{Gbit} / \mathrm{s}$ receiver we used for the BER measurements, which has a much lower bandwidth than that of the timing jitter we introduce in the pulses. For this reason, we also examined timing jitter and amplitude noise distributions of the data from the eye diagrams for all the cases discussed previously. Fig. 3a shows the standard deviation of the timing jitter before and after our system for various values of the induced timing jitter, as measured at the full width at half maximum point on the leading edge of the pulses. At the input of our system the rms timing jitter of the pulses increases linearly with induced timing jitter values. At the system output these values remain fairly constant and approach the original value of the clock signal ( $\sim 0.9 \mathrm{ps}$ as measured with the same technique). We also examined histogram data on pulse amplitude noise, as measured at the pulse centre, for various noise settings in the system. Q-factor values for these cases are shown in Fig.3b. A Q-factor increase from 5.9 to 8 was measured in the worst condition of $\sim 11 \mathrm{ps}$ induced timing jitter.
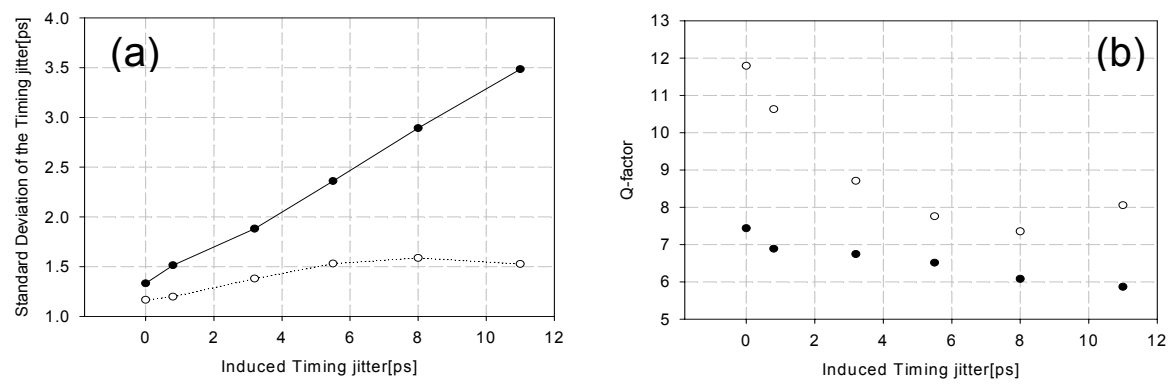

Figure 3a: Timing jitter standard deviations versus induced timing jitter characteristic before (full-circles) and after (open-circles) our system. 3 b: Q-factor values versus induced timing jitter before (full-circles) and after (open-circles) our system

\section{Conclusion}

We have used the combination of a rectangular-pulse SSFBG and a NOLM to temporally expand and flatten the noisy data pulses prior to switching them with clean clock pulses. The linear reshaping function of the SSFBG is responsible for eliminating any mistiming of the pulses at the switch output, while by using the data as the control signal to the NOLM we are able to equalize the pulse amplitudes of the switched output signal. We have demonstrated the technique at $10 \mathrm{Gbit} / \mathrm{s}$, we envisage though that this scheme will be useful in ultra-high bit rate communications.

\section{References}

1. P. Petropoulos, M. Ibsen, A. D. Ellis, and D. J. Richardson, "Rectangular pulse generation based on pulse reshaping using a superstructured fiber Bragg grating", J. of Lightwave Technol.,. 19, 746-752 (2001).

2. J. H. Lee, P. C. Teh, P. Petropoulos, M. Ibsen, and D. J. Richardson, "Timing jitter tolerant all-optical modulator and demultiplexing systems incorporating pulse-shaping fiber Bragg gratings", in Proc. OFC, Postdeadline paper PD30 (Anaheim, California 2001).

3. J.H.Lee, L.K.Oxenlowe, M.Ibsen, K.S.Berg, A.T.Clausen, D.J.Richardson, P.Jeppesen, "Timing jitter tolerant all-optical TDM data demultiplexing at $80 \mathrm{Gbit} / \mathrm{s}$ using fiber Bragg grating based rectangular pulse switching technology", in Proc. OFC, OSA Technical Digest, vol. I, 200-201 (Atlanta, Georgia, 2003).

4. F. Parmigiani, P. Petropoulos, M. Ibsen,. B. C. Thomsen, and D. J. Richardson, "Retiming of short optical pulses using linear pulse reshaping and all-optical switching”, ”, in Proc. OFC, OSA Technical Digest, , Paper FC7 (Los Angeles, California, 2004).

5. N.J Doran, and D. Wood, "Nonlinear-optical loop mirror" Opt.Lett. 13, 56 (1988). 